\title{
Switching modes in easy and hard axis magnetic reversal in a self-assembled antidot array
}

\author{
Felix Haering ${ }^{1}$, Ulf Wiedwald ${ }^{1,5}$, Steffen Nothelfer $^{1}$, Berndt Koslowski ${ }^{1}$, \\ Paul Ziemann $^{1}$, Lorenz Lechner ${ }^{2}$, Andreas Wallucks ${ }^{3}$, Kristof Lebecki ${ }^{3}$, \\ Ulrich Nowak $^{3}$, Joachim Gräfe ${ }^{4}$, Eberhard Goering ${ }^{4}$ and Gisela Schütz ${ }^{4}$ \\ ${ }^{1}$ Institute of Solid State Physics, Ulm University, Albert-Einstein-Allee 11, D-89069 Ulm, Germany \\ ${ }^{2}$ Electron Microscopy Group of Materials Science, Ulm University, Albert-Einstein-Allee 11, D-89069 \\ Ulm, Germany \\ ${ }^{3}$ Department of Physics, University of Konstanz, D-78457 Konstanz, Germany \\ ${ }^{4}$ Max Planck Institute for Intelligent Systems, Heisenbergstrasse 3, D-70569 Stuttgart, Germany
}

E-mail: ulf.wiedwald@uni-due.de

\begin{abstract}
We study the reversal mechanisms in a self-assembled, hexagonally ordered Fe antidot array with a period of $200 \mathrm{~nm}$ and an antidot diameter of $100 \mathrm{~nm}$ which was prepared by polystyrene nanosphere lithography. Direction-dependent information in such a self-assembled sample is obtained by measuring the anisotropic magnetoresistance (AMR) through constrictions processed by focused ion beam milling in nearest neighbor and next nearest neighbor directions. We show that such an originally integral method can be used to investigate the strong in-plane anisotropy introduced by the antidot lattice. The easy and hard axis reversal mechanisms and corresponding AMR signals are modeled by micromagnetic simulations. Additional in-field magnetic force microscopy studies allow the correlation of microscopic switching to features in the integral AMR. We find that the easy axis of magnetization is connected to a distinct periodic magnetic domain pattern, which can be observed during the whole magnetization reversal. While this process is driven by nucleation and propagation of reversed domains, the hard axis reversal is characterized by a (stepwise) rotation of the magnetization via the antidot lattice' easy axes.
\end{abstract}

\section{Introduction}

Introducing periodic arrays of holes-so-called antidotsinto magnetic thin films greatly alters their magnetic properties. Shape anisotropy and domain wall pinning lead to enhanced coercive fields and to distinct magnetic domain configurations [1]. It has been shown that different aspects of the magnetic behavior [2] as well as magneto-optical properties [3] can be influenced and, thus, optimized by tuning the geometrical parameters of antidot arrays. Possible applications reach from magnetic data storage media [4] to

\footnotetext{
5 New address: University of Duisburg-Essen, Faculty of Physics, D-47057
} Duisburg, Germany. the creation of artificial spin-ice structures $[5,6]$ and the emerging field of magnonics, where spin waves are used and manipulated to transport and process information [7-9].

A commonly used approach to create such antidot structures on the nanoscale is colloidal lithography [10-13]. As compared to e-beam lithography, this is a cost-efficient, easily scalable and variable technique. It suffers, however, from a lack of long range structural order of antidots which leads to orientational averaging and prevents extraction of any anisotropic behavior of magnetic properties. This is particularly true when the length scale of the antidot array is reduced to characteristic length scales of the magnetic material such as the domain wall width. With these parameters 
being in the range of $\sim 100 \mathrm{~nm}$, the fabrication of the antidot arrays as well as the analysis of their magnetic properties constitute a significant experimental challenge.

In this contribution we present an easily scalable method based on colloidal lithography which makes such direction-dependent information accessible by means of magnetotransport measurements even within truly nanoscaled antidot arrays. Unlike in previous publications [14], we use the anisotropic magnetoresistance [15] as a local probe of the sample's magnetization in a single crystallite of the self-assembled antidot array which has been selected by focused ion beam (FIB) milling. This approach allows identification of the easy and hard axis of the magnetization reversal. Application of in-field magnetic force and Kerrmicroscopy allows the correlation of distinct features in the AMR measurements with the sample's actual magnetization configuration. Additionally, we conduct micromagnetic simulations of both, the easy and hard axis magnetization reversal and derived the corresponding AMR signal to enhance our interpretation of experimental results [16].

\section{Experimental details}

The studied antidot arrays are produced by colloidal lithography [17]. Close-packed monolayers of commercial polystyrene (PS) nanospheres (Invitrogen) with a mean diameter of $205 \pm 8 \mathrm{~nm}$ are deposited on $\mathrm{SiO}_{2}(300 \mathrm{~nm}) / \mathrm{Si}(001)$ substrates by dip coating at an extraction velocity of $10 \mu \mathrm{m} \mathrm{s}^{-1}$ and an angle of $60^{\circ}$ between the substrate plane and the air-water interface. Details of this process can be found elsewhere [6]. After self-assembly of PS spheres, their diameter is reduced to $100 \pm 8 \mathrm{~nm}$ by exposing the sample to oxygen plasma (DC bias: $-85 \mathrm{~V}$ ) for $120 \mathrm{~s}$. We have previously demonstrated that the spherical shape of the PS spheres can be maintained throughout the etching process applied [17, 18]. On top of this template we grow a $20 \mathrm{~nm}$ thick Fe film and a $2 \mathrm{~nm} \mathrm{Pt}$ capping layer for oxidation protection through a rectangular mask by pulsed laser deposition under UHV conditions $[18,19]$. The resulting antidot sample has a length of $6 \mathrm{~mm}$ and a width of $0.5 \mathrm{~mm}$ (red bar in figure 1). Subsequently, PS spheres together with their magnetic caps were removed by chemo-mechanical polishing. Finally, additional $\mathrm{Pt}$ contacts $(50 \mathrm{~nm})$ are deposited by PLD for transport measurements (yellow pads in figure 1). The chosen setup allows performing two independent 4-point-resistance measurements simultaneously, with the middle contacts separating the sample in halves.

The self-assembly approach is fast and reliable. However, the crystallites of the resulting antidot array, i.e. areas of constant antidot lattice orientation, are typically restricted to a size of $20 \times 20 \mu \mathrm{m}^{2}$. This implies that the two sample halves contain a multitude of such domains with all possible lattice orientations. Thus, any magnetotransport measurement would average over all orientations of antidot crystallites making any detection of anisotropic in-plane magnetization reversal inaccessible $[13,20]$. We circumvent this by milling constrictions in nearly perfectly ordered regions using $\mathrm{Ga}^{+}$ ions in a focused ion beam (FIB) machine (Zeiss NVision

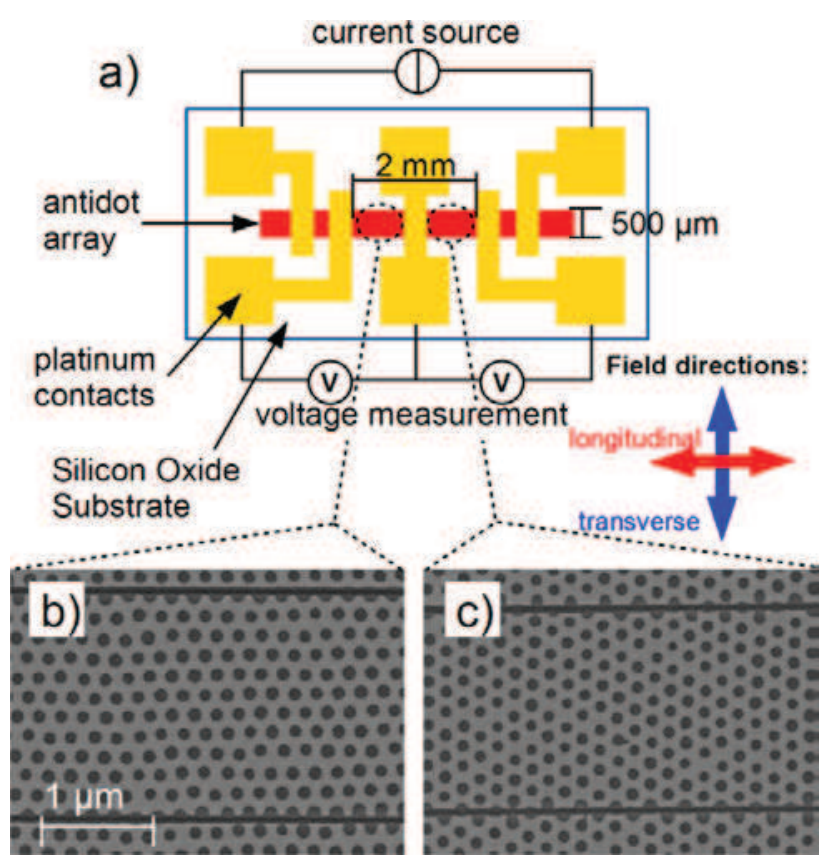

Figure 1. (a) Schematics of the sample geometry for AMR measurements. The red and blue arrows indicate the direction of the magnetic field for longitudinal and transverse geometries, respectively. SEM images show antidot arrays with FIB-milled cuts (dark lines) along the nearest neighbor (b) and along the next nearest neighbor direction (c).

40 SEM/FIB). Therefore we searched for crystallites of suitable size and antidot orientation, i.e. either the nearest neighbor (nn) direction or the next nearest neighbor (nnn) direction aligned parallel to the net current direction in AMR measurements (cf figure 1). The cuttings create two channels, each having a length of $20 \mu \mathrm{m}$ and width of approximately $2.5 \mu \mathrm{m}$, thus each enveloping more than 1000 well-ordered antidots. Parts of the two resulting antidot arrays with cuts along nn- and nnn-directions parallel to the current are shown in figures 1(b) and (c), respectively.

Magnetoresistance measurements were performed using a Keithley 6221 as a DC current source, applying a maximum probing current of $10 \mu \mathrm{A}$, and two Keithley 2000 digital multimeters as sketched in figure 1 . Temperature stability is provided by using a liquid nitrogen bath cryostat $(T=77 \mathrm{~K})$. This is necessary as the AMR effect in the Fe antidot array is small $(\sim 0.1 \%)$, thus even minor changes in temperature would obscure the signal.

The FIB-processing has the desired effect on the electrical resistance: due to the small width and sufficient length $\left(2.5 \times 20 \mu \mathrm{m}^{2}\right.$ compared to pre-cut dimensions of $1 \times 0.5 \mathrm{~mm}^{2}$ ) of the FIB-cut channels, the electrical resistance of each half of the sample is increased to about $420 \Omega$ as compared to a value of $68 \Omega$ prior to cutting. Thus, more than $80 \%$ of the magnetotransport signal originate from FIB-cut regions. The setup is capable of magnetic fields up to 5000 Oe applied in any direction with respect to the sample. Spatially and angularly resolved MOKE magnetometry are measured on the FIB channels using a Durham Magneto Optics NanoMOKE3 magnetometer within a laser focus of about $2 \mu \mathrm{m}$. 
cut in $n n$ direction:
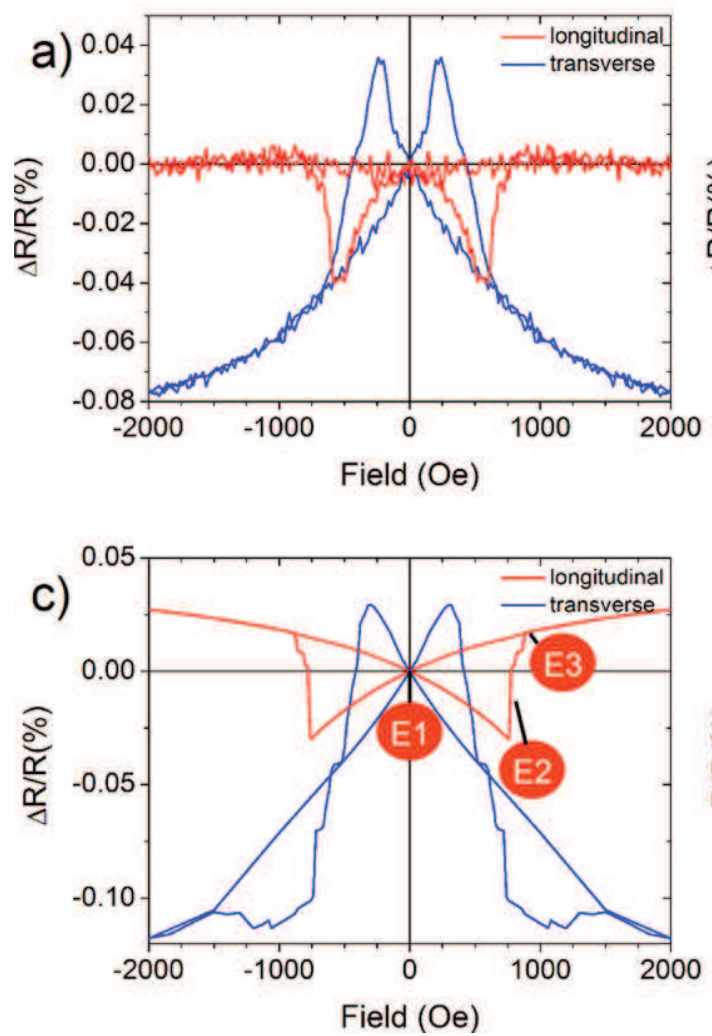

cut in nnn direction:
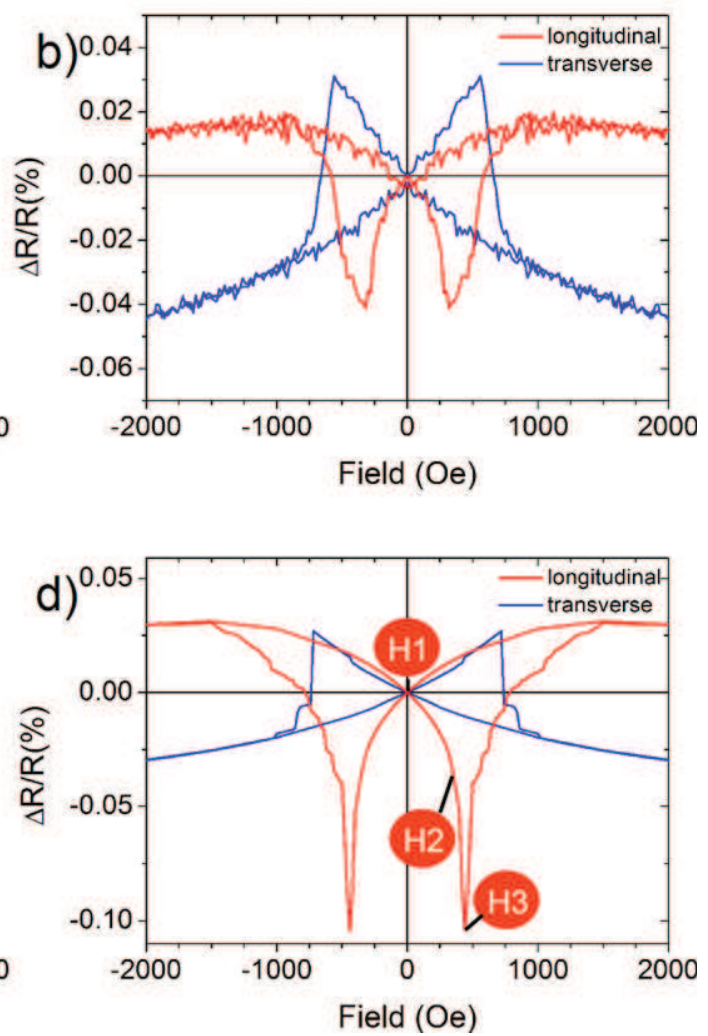

Figure 2. Measured AMR curves (a) for the current direction along nearest neighbors (nn) and (b) next nearest neighbor directions (nnn) in longitudinal (red) and transverse (blue) geometry of the external field at $T=77 \mathrm{~K}$. Panels (c) and (d) present micromagnetic simulations of the corresponding geometries in identical color code. The red indicators in panel (c) and (d) mark significant points of AMR curves for easy (E) and hard axis $(\mathrm{H})$ magnetic switching as shown in figures 3 and 4.

Magnetic force microscopy (MFM) was carried out using a home-built AFM described in [21] in conjunction with Bruker MESP magnetic tips. Additionally, an electro-magnet capable of in-plane magnetic fields of up to 1000 Oe with yoke size of $3 \mathrm{~mm}$ was fitted to the instrument. However, it is worth mentioning that the MFM data presented in this work are not recorded from the FIB patterned regions but from a reference sample to fit into the yoke of the magnet.

\section{Setup of micromagnetic simulations}

Micromagnetic simulations are carried out using the OOMMF package [22]. The channels along nn- and nnn-directions are modeled as bars with a length of $8 \mu \mathrm{m}$, width of $2 \mu \mathrm{m}$ and thickness of $20 \mathrm{~nm}$. We use the bulk magnetic properties of $\mathrm{Fe}$ : saturation magnetization $1.7 \mathrm{MA} \mathrm{m}^{-1}$, exchange constant $21 \mathrm{pJ} \mathrm{m}^{-1}$ and cubic anisotropy constant $48 \mathrm{~kJ} \mathrm{~m}^{-3}$. The polycrystallinity of the Fe films is incorporated by random anisotropy axes for each anisotropy crystallite. These crystallites are modeled as rectangular prisms, each having a size of $10 \times 10 \times 20 \mathrm{~nm}^{3}$. The mesh size $\left(2 \times 2 \times 10 \mathrm{~nm}^{3}\right.$, e.g. two layers in the out-of-plane direction) is chosen to avoid large angles between magnetization vectors in neighboring cells [23]. The experimentally observed non-perfect antidot structure is mimicked by arbitrary variations of antidot diameters and positions of $\pm 5 \%$. Although the AMR signal is measured only on the $2 \times 20 \mu \mathrm{m}^{2}$ bar, the magnetization within this area is influenced by the whole millimeter-long and wide sample, namely via long range dipolar interactions. This can be seen from the results in figure 2, where no extra shape anisotropy rising from the FIB-cut edges can be observed. To include this finding in our simulations we have added an additional 'artificial' uniaxial anisotropy that cancels out the $8 \times 2 \mu \mathrm{m}^{2}$ shape anisotropy.

Domain nucleation plays an important role during the reversal process. The sample edge roughness is taken into account: simulated long edges $(8 \mu \mathrm{m}$ long) are not perfect, the upper edge is placed roughly in the middle of the holes, while we positioned the lower one roughly between two rows of antidots as can be seen in figure 3(b). Thus, all possible nucleation scenarios are taken into account. Experimental results are directly affected by domain nucleation on the long edges, but also indirectly by reversal processes that might appear somewhere to the left and to the right of the measured $20 \mu \mathrm{m}$ long bar. To consider this, the presented magnetotransport curves are calculated only from the central simulated square of an area of $2 \times 2 \mu \mathrm{m}^{2}$. With this arrangement we model the direct influence of the domain nucleation at the upper and the lower edge and indirect influence of the nucleations from the left and from the right.

To calculate the AMR signal from the simulated magnetization we perform the following procedure which has 
a)
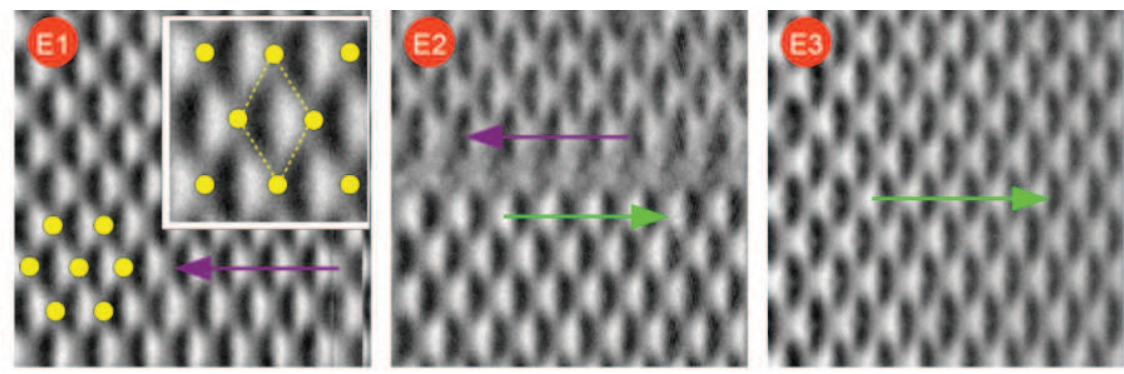

b)
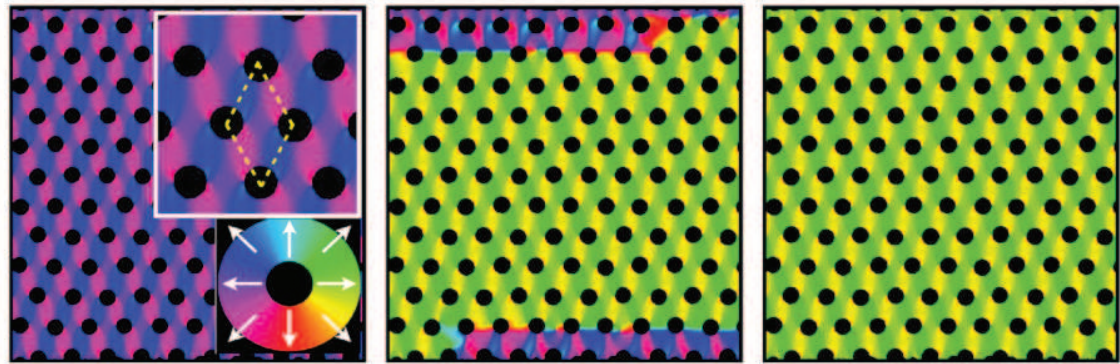

c)
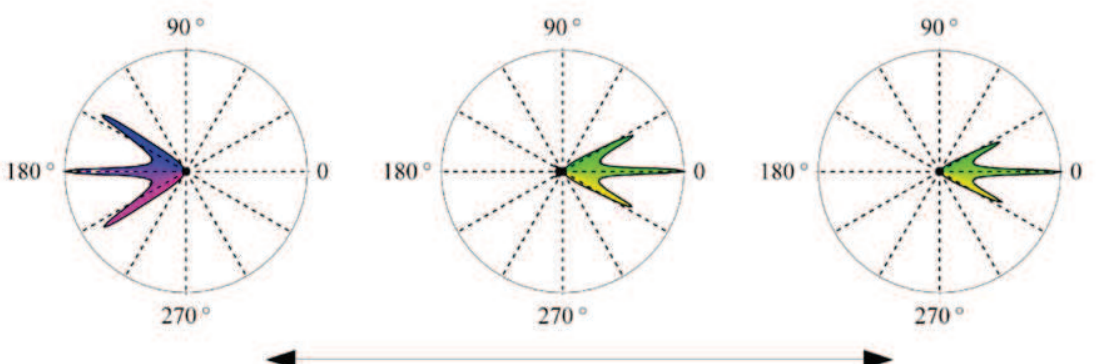

\section{Magnetic Field Direction}

Figure 3. Easy axis magnetization reversal. (a) MFM images taken at the positions marked in figure 2(c). The colored arrows indicate the local average magnetization direction. The insets give a magnified view with the dashed lines referring to the antidot lattice unit cell.

(b) Micromagnetic configuration obtained from the simulations. The histograms in (c) show the angular orientation of magnetization for the snapshots of row (b).

been described in more detail in [24]. As a result of the antidot pattern, the current density distribution is inhomogeneous. In a first step, we calculated this two-dimensional vector field $j_{0}(r)$ using the open source software IDC2D [25]. We stress that this is done independently from the applied field or magnetization configuration, i.e. resistivity is considered to be isotropic at this stage. In the second step we consider this current distribution as well as the magnetization vector (separately for every applied field), and we calculate local anisotropic magnetoresistance: $r_{\mathrm{AMR}}(r)=\Delta R_{\max } \sin ^{2} \vartheta(r)=$ $\sigma_{\mathrm{AMR}}(r)^{-1}$ with $\vartheta$ being the angle between the current density and the magnetization (see below for the explanation of $\left.\Delta R_{\max }\right)$. In the third step, the total current density is calculated, $j_{\text {tot }}(r)=\sigma_{\mathrm{AMR}}(r) E_{0}+j_{0}$, where $E_{0}$ is the electrical field, which is not influenced by the AMR effect. This approximation is valid since the changes in resistivity due to magnetoresistance are very small. The fourth step consists of integrating the current density $j_{\text {tot }}$ over a cross section in order to get the total current $I_{\text {tot }}$. In the last step the total resistivity together with the AMR contribution, was deduced using $I_{\text {tot }}$, the applied voltage and Ohms law. The whole procedure, together with its approximations have been described and tested in [24] — there the reader can find also other, possible approaches.

\section{Results and discussion}

The experimental longitudinal and transverse AMR measurements at $T=77 \mathrm{~K}$ for both the channels cut along $\mathrm{nn}$ - and nnn-directions are shown in figures 2(a) and (b), respectively. All curves are normalized to the resistance $R_{0}$ of the remanent state. As expected, the longitudinal curves (red) exhibit characteristic dips while we find peaks in the transverse geometry (blue) as observed in many experiments before $[26,27]$. In an experiment prior to the FIB-cutting, we compared AMR curves of both longitudinal and transversal geometry with the hysteresis loop obtained by SQUID magnetometry and found that dips and peaks occur in the direct vicinity of the coercive field $H_{\mathrm{C}}=380$ Oe. Here, the first important result shows up: the position of the peaks (dips) and, thus, the coercive field strongly depends on the field direction, indicating that our approach is suitable of resolving the in-plane anisotropy introduced by the antidot lattice. For example, when FIB-cutting is applied along the 
nn-direction (figure 2(a)) dips appear at 570 Oe while peaks are observed at 240 Oe. This splitting of the coercive field can be directly linked to the relative orientation of the external magnetic field with respect to the orientation of the antidot lattice. For the channel cut in nn-direction, the longitudinal field orientation corresponds to the easy axis of the sample. When we switch to transverse geometry, we consequently apply the field parallel to the nnn-direction due to the hexagonal symmetry of antidots (cf figure 1) being the hard axis of magnetization. Furthermore, we can exclude that the observed shift in coercive fields is due to the channel's macroscopic shape. In that case, the easy axis always would be expected parallel to the long side of the stripe. Contrary to this expectation, for the channel cut along the nnn-direction the higher coercive field $(580 \mathrm{Oe})$ is measured with the field applied perpendicular to the cut direction, thus again along a nn-direction of the antidots (transverse AMR curve in figure 2(b)). This finding is also supported by angular-resolved MOKE measurements performed on the respective channels, where a 6-fold symmetry of the coercive field is resolved (not shown).

The corresponding micromagnetic simulations of the AMR-experiments displayed in figures 2(c) and (d) show remarkable agreement with the experimentally observations in terms of the overall shape, the peak (dip) positions and their magnitude for all four experimental geometries.

For the detailed discussion of switching modes we restrict ourselves to the simulations and experiments in longitudinal geometry. Discussion of the transversal behavior follows similar arguments. Inspection of the simulations in figures 2(c) and (d) suggests that there are two distinct magnetization reversal mechanisms, depending on whether the field is applied along the nn or nnn-direction which correspond to the easy axis and hard axis, respectively. Some outstanding magnetic states in figures 2(c) and (d) are indicated and will be referred to by the assigned labels 'easy' (E) and 'hard' (H) in the following. Figures 3(a) and 4(a) show MFM results of the indicated states and the corresponding simulated magnetization patterns are presented in figures 3(b) and 4(b). Additionally, the angular distributions of spins were extracted from the simulations and can be found in figures 3(c) and 4(c) for easy and hard axis reversal, respectively.

First, we discuss the easy axis reversal mode, i.e. the field is applied in nn-direction. The remanent state (E1) is given by a periodic magnetization pattern in which each unit cell consists of five domains: one central domain with a magnetization pointing towards the nearest neighbor direction and four domains at an angle of $\pm 30^{\circ}$ with respect to the direction of the initially applied field $[6,28,29]$. In the spin angular distribution histograms (figure 3(c)) this is reflected by the occurrence of three peaks at $180^{\circ}, 150^{\circ}$ and $210^{\circ}$. This configuration is rather stable as implied by the high coercive field and is connected to a high resistance state in the AMR curve. This is expected considering the general AMR relation [15] $R=R_{\mathrm{II}}-\Delta R_{\max } \sin ^{2} \vartheta$ with $\vartheta$ indicating the angle between current density and applied magnetization leading to $R_{\perp}=R_{\mathrm{II}}-\Delta R_{\max }$ for magnetizations approaching saturation. In MFM (figure 3(a)), a periodic arrangement of rhombi can be seen in this state. The yellow dots give the antidot center positions and make clear that each rhombus belongs to a unit cell of the antidot lattice. The mirror axis of the rhombi (perpendicular to the black-white contrast) is a clear indication of the magnetization direction [6]-in the $E 1$ state parallel to the initially negative saturating field, as illustrated by the red arrow in figure 3(a).

An increasing positive reversal field applied $(\mathrm{E} 1 \rightarrow \mathrm{E} 2)$ in $0^{\circ}$-direction results in a torque acting on the four tilted domains which initially enclosed an angle of $\pm 30^{\circ}$ with the external field, giving rise to larger angles between magnetization and the applied current, which is seen in the AMR measurement as the resistance drops steadily in accordance with the above angular dependence. Close to the coercive field, the stable domain structure breaks down (E2): in MFM (figure 3(a)) as well as in the simulations (figure 3(b)) reversed mesoscopic domains have nucleated and grown. Especially in MFM it is obvious that the local average magnetization points either in the original direction or is reversed by $180^{\circ}$. These larger domains are separated by a domain wall with a width of one antidot lattice constant. For the AMR effect with its $\sin ^{2} \theta$-dependence, both magnetization directions are almost equivalent (neglecting torque on the off-field domains caused by the applied magnetic field), which explains the comparatively small change of the AMR signal in this geometry. The switching is complete when the original mesoscopic domain is no longer visible since completely reversed. In that case, again a strictly periodic magnetization pattern is obtained (E3), which is, however, inverse to the starting condition (E1). Finally, still larger external fields turn the four side domains in the direction of the external field and saturate the sample.

More interesting is the switching mode in nnn-direction (hard axis reversal). It is characterized by a rotation of the magnetization via intermediate nearby easy axes: as the field is reduced in the hard axis (H1), the magnetization relaxes again in a state very similar to the one obtained for the easy axis, the whole pattern, however, is rotated by $30^{\circ}$ with respect to the direction of the initial field (figures 4(a) and (b)), i.e. the central domain aligns to an easy direction (figure 4(c)). Whether the tilt is $+30^{\circ}$ or $-30^{\circ}$ is determined by defects in the antidot lattice arrangement or by a slight misalignment of the external field with respect to the nn-direction-in fact one can observe rotations in both directions in the simulations (we observe five peaks instead of three in the corresponding spin angular distribution) while the rotation in MFM-experiments appears almost coherent.

Application of a counter-field turns the magnetization out of the easy axis alignment (H2), as becomes clearly visible in figure 4(c) where the average magnetization is found to be tilted by approximately $10^{\circ}$ with respect to the remanent state (H1). In the corresponding MFM image (figure 4(a)) this tilt of the magnetization can be seen as the symmetry of the rhombi is broken, which becomes clearer by comparing the insets of the MFM images of states H1 and H2. Furthermore, the rotation is no longer coherent: While the majority of the investigated sample area still exhibits a mean magnetization close to the $150^{\circ}$ easy axis (blue arrow), the magnetization 
a)

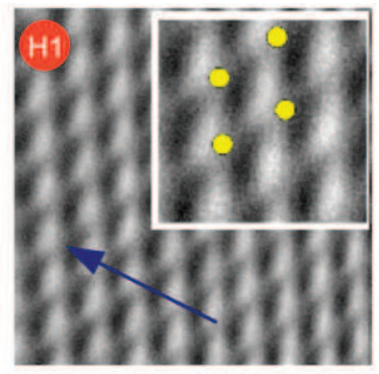

b)

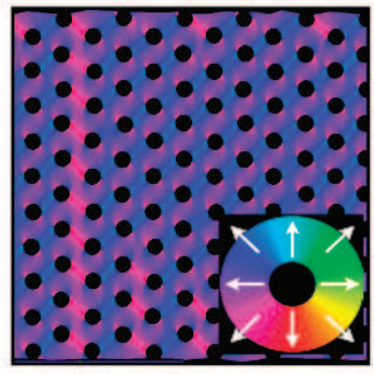



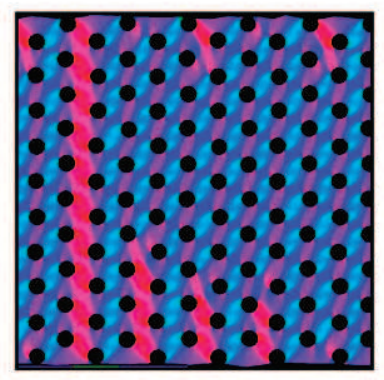

c)

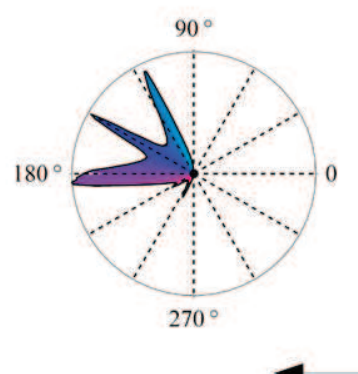



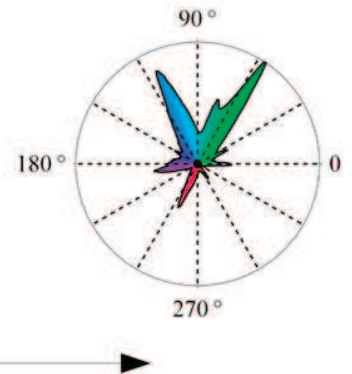

$90^{\circ}$

Magnetic Field Direction

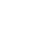
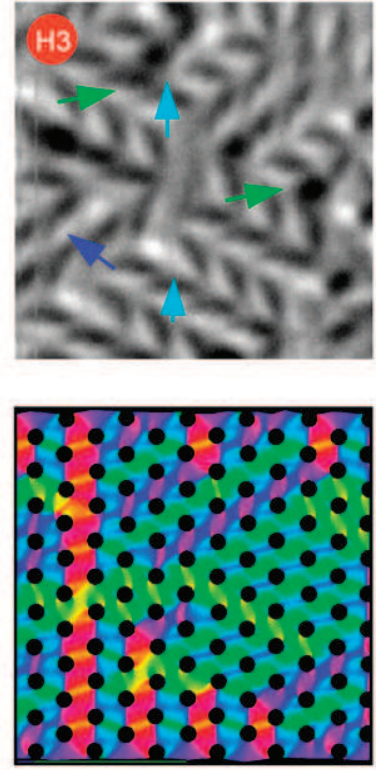


Figure 4. Hard axis magnetization reversal. (a) MFM images taken at the positions marked in figure 2(d). The colored arrows indicate the local mean magnetization direction. The insets give a magnified view. (b) Micromagnetic configuration obtained from the simulations. The histograms in (c) show the angular orientation of magnetization for the snapshots of row (b). (d) Hysteresis Loops with the field applied along the next nearest neighbor direction measured by KERR-Microscopy. The red curve shows the projection of the magnetization on the field direction, the blue curve represents the projection on the in-plane direction perpendicular to the applied field. (e) shows the corresponding curves from the simulations.

within a small stripe rotated into the $210^{\circ}$ easy axis (pink arrow).

At the coercive field (state $\mathrm{H} 3$ ), both experiment (figure 4(a)) and simulations (figure 4(b)) suggest that the magnetization splits into a multitude of mesoscopic domains, all of which showing a mean magnetization pointing close to one of the antidot lattice' easy axis. In MFM this is once again seen by means of the rhombi. Their symmetry axes and, thus, their mean magnetization points towards the $150^{\circ}$ (dark blue arrow), $90^{\circ}$ (teal arrows) as well as the $30^{\circ}$ (green arrows) easy axis. In the spin angular distributions derived from the simulations one can observe three major peaks at $130^{\circ}, 100^{\circ}$ and $65^{\circ}$. These indicate that the majority of the sample's magnetization is in the $90^{\circ}$ easy axis configuration which is distorted towards higher angles by the presence of the external field. In this configuration the largest angles between the magnetization and the current flow directions are obtained, resulting in the minimum in the AMR curve. Increasing the 
counter-field further rotates the majority of the magnetization into the $30^{\circ}$ easy axis configuration and eventually saturates the sample along the $0^{\circ}$ direction (not shown).

Such a successive occupation of the easy axis $\left(150^{\circ} \rightarrow\right.$ $90^{\circ} \rightarrow 30^{\circ}$ ) in the course of the magnetization reversal was also found in the work of Manzin et al [30], where hexagonal permalloy antidot arrays were studied by micromagnetic simulations under application of periodic boundary conditions. However, this kind of idealization leads to a fully collective rotation of the magnetization without the formation of mesoscopic domains, which we could find in our experiment.

Many features of the reversal process discussed here were also found in the investigation of epitaxial bcc Fe thin films on GaAs by $\mathrm{Gu}$ et al [31], where for the field swept along the [110] crystallographic direction (hard axis) the magnetization also relaxes coherently into one of the neighboring easy axis (e.g. [100] direction) at first, similarly followed by nucleation of domains occupying the easy axis enclosing smaller angles with the subsequently applied reversal field. While in case of the epitaxial film the mechanism is determined by the magnetocrystalline anisotropy, in case of the antidot array this is replaced by a periodic modulation of shape anisotropy, once again underlining the analogy between phenomena on the atomic and the mesoscopic length scale.

The rotational reversal mechanism also shows a distinct signature in MOKE-Microscopy, which we applied on the channel in nnn-direction with the field direction parallel to the channel. Figure 4(d) presents the magnetic hysteresis loops projected on the field axis (red) and perpendicular to the field axis (blue), while figure 4(e) is the result from the corresponding simulation. As the external field is reduced, an off-field component is detected, indicating that the overall magnetization begins to rotate as predicted by the simulations (H1). Further increase of the counter-field increases this off-field component (H2) until it reaches a peak (H3). For larger external fields the off-axis component vanishes continuously as the magnetization approaches saturation.

\section{Conclusions}

In summary, we have prepared hexagonally ordered magnetic antidot arrays on the nanoscale and investigated the magnetic switching modes in nearest neighbor (nn) and next nearest neighbor (nnn) directions by AMR, MFM and MOKEMicroscopy and related micromagnetic simulations. By FIBcutting we modified our previously integral magnetotransport measurement into a local probe for the magnetization in a single crystallite of the self-assembled antidot lattice. These crystallites exhibit the expected 6-fold symmetry induced by the hexagonally ordered antidots. Micromagnetic simulations and MFM showed two distinct reversal modes: In the nn-direction (easy axis) the sample's magnetization is stable up to a field leading to the nucleation and growth of $180^{\circ}$ reversed domains. In the nnn-direction (hard axis), however, the switching mode differs significantly. In this case micromagnetic simulations suggest a reversal mode in which the mean magnetization jumps from one easy direction to the next until the magnetization is driven in the direction of the external field. Contrary to previous results from simulations using periodic boundary conditions [30], where this rotation happens coherently, in our case the sample splits up into smaller domains. In additional experiments by MOKE-microscopy such behavior has clearly been proven by a peak in the off-axis magnetization component.

\section{Acknowledgments}

We thank Pavan Muralidhar, Fabian Enderle, Stefan Wiedemann and Jörg Bernhard for the assistance in sample preparation and Karsten Küpper for valuable discussions.

Financial support by the Baden-Württemberg Stiftung is gratefully acknowledged.

\section{References}

[1] Heyderman L J et al 2006 Phys. Rev. B 73214429

[2] Wang C C, Adeyeye A O and Singh N 2006 Nanotechnology 171629

[3] Papaioannou E Th, Kapaklis V, Patoka P, Giersig M, Fumagalli P, Garcia-Martin A and Ferreiro-Vila E 2010 Phys. Rev. B 81054424

[4] Jalil M B A 2003 J. Appl. Phys. 937053

[5] Mengotti E, Heyderman L J, Fraile Rodriguez A, Nolting F, Hügli R V and Braun H-B 2011 Nature Phys. 7 68-74

[6] Haering F, Wiedwald U, Häberle T, Han L, Plettl A, Koslowski B and Ziemann P 2013 Nanotechnology 24055305

[7] Zivieri R et al 2012 Phys. Rev. B 85012403

[8] Kruglyak V V, Demokritov S O and Grundler D 2010 J. Phys. D: Appl. Phys. 43264001

[9] Lenk B, Ulrichs H, Garbs F and Münzenberg M 2011 Phys. Rep. 507107

[10] Kim J, Cho S-G, Choa Y-H, Shin G J and Kim J 2012 Thin Solid Films 520 3013-6

[11] Tiberto P, Boarino L, Celegato F, Barrera G, De Leo N, Coisson M, Vinai F and Allia P 2012 Appl. Surf. Sci. 25944

[12] Zhukov A A, Goncharov A V, de Groot P A J, Bartlett P N and Ghanem M A 2003 J. Appl. Phys. 937322

[13] Ho C-C, Hsieh T-W, Kung H-H, Juan W T, Lin K-H and Lee W-L 2010 Appl. Phys. Lett. 96122504

[14] Castano F J, Nielsch K, Ross C A, Robinson J W A and Krishnan R 2004 Appl. Phys. Lett. 852872

[15] McGuire T R and Potter R I 1975 IEEE Trans. Magn. 111018

[16] Miltat J and Donahue M J 2007 Handbook of Magnetism and Advanced Magnetic Materials vol 2, ed H Kronmüller and S S P Parkin (Chichester: Wiley-Interscience) p 742

[17] Plettl A, Enderle F, Saitner M, Manzke A, Pfahler C, Wiedemann S and Ziemann P 2009 Adv. Funct. Mater. 193279

[18] Wiedwald U et al 2012 Beilstein J. Nanotechnol. 3831

[19] Trautvetter M, Wiedwald U, Paul H, Minkow A and Ziemann P 2011 Appl. Phys. A 102725

[20] Tiberto P, Boarino L, Celegato F, Coïsson M, De Leo N, Vinai F and Allia P 2010 J. Appl. Phys. $10709 B 502$

[21] Häberle T, Haering F, Pfeifer H, Han L, Kuerbanjiang B, Wiedwald U, Herr U and Koslowski B 2012 New J. Phys. 14043044

[22] Donahue M J and Porter D G 2012 http://math.nist.gov/oommf

[23] Lebecki K M, Hinzke D, Nowak U and Chubykalo-Fesenko O 2012 Phys. Rev. B 8694409 
[24] Wallucks A 2012 Computer simulations on the magnetoresistance in antidot lattices Bachelor Thesis University of Konstanz (see: http://books.google.pl/books? id=1WF7MwEACAAJ)

[25] http://idc2d.sourceforge.net/

[26] Wang C C, Adeyeye A O, Singh N, Huang Y S and Wu Y H 2005 Phys. Rev. B 72174426

[27] Meng T J, Laloe J-B, Holmes S N, Husmann A and Jones G A C 2009 J. Appl. Phys. 106033901
[28] Ctistis G, Papaioannou E, Patoka P, Gutek J, Fumagalli P and Giersig M 2009 Nano Lett. 91

[29] Béron F, Pirota K R, Vega V, Prida V M, Fernández A, Hernando B and Knobel M 2011 New J. Phys. 13013035

[30] Manzin A and Bottoauscio O 2012 J. Phys. D: Appl. Phys. 45095001

[31] Gu E, Bland J A C, Daboo C, Gester M, Brown L M, Ploessl R and Chapman J N 1995 Phys. Rev. B 513596 